\title{
Malattia sporotricoide da M. marinum in un soggetto immunocompetente
}

\author{
Carmelo Mangano', Nicola Foti', Maria Stella Carpentieri', Saverio De Lorenzo ${ }^{2}$, \\ Paolina Cavalcanti ${ }^{3}$, Maria Agnese Filia $^{3}$, Anna Maria Cannataro ${ }^{3}$, Saveria Dodaro ${ }^{3}$, \\ Luigi Maria Greco ${ }^{3}$, Alfredo Vallone ${ }^{4}$
}

\author{
' Malattie Infettive, Azienda Ospedaliera, Reggio Calabria \\ ${ }^{2}$ Scuola di Specializzazione in Malattie Infettive, Università degli Studi, Messina \\ ${ }^{3}$ Servizio di Virologia e Microbiologia, Presidio Ospedaliero Annunziata, Cosenza \\ ${ }^{4}$ Reparto di Malattie Infettive, Presidio Ospedaliero Annunziata, Cosenza
}

\section{INTRODUZIONE}

Molti dei "micobatteri atipici" cioè diversi da quelli che causano la tubercolosi, definiti dagli anglosassoni Nontuberculous Mycobacteria (NTM) sono responsabili di infezioni localizzate della cute e dei tessuti sottocutanei in ospiti immunocompetenti (9). Comunque, in termini di patogenicità emergente, non mancano segnalazioni di casi con infezioni disseminate e/o a localizzazione polmonare in ospiti immunocompromessi e con malattia HIV correlata, oltre a quelle ad esclusiva localizzazione cutanea, in pazienti HIV con linfoma e leucemia (3) e miastenia grave (5). Tali agenti sono elencati nella classificazione di TIMPE e RUNYON a seconda delle loro caratteristiche distintive di specie.

Le sindromi cliniche associate a tali micobatteri sono identificabili come:

$>$ Lesioni granulomatose (granulomi delle piscine) da M. marinum.

$>$ Malattia Sporotricoide da M. marinum, più frequentemente, e meno da $M$. fortuitum, $M$. chelonae (abscessus), M. kansasii e M. haemophilum.

$>$ Ascessi, ulcere e fistole da M. fortuitum e $M$. abscessus.

$>$ Ulcere necrotiche croniche (ulcera di Buruli) da M. ulcerans.

Ciò nondimeno, anche se sembra che ogni specie di NTM possa essere in pratica causa di malattia cutanea (2) i più frequenti agenti responsabili restano M. fortuitum, M. abscessus, M. ulcerans e M. marinum.

Questi agenti, tutti patogeni per l'uomo, hanno come serbatoio l'acqua e la fauna marina. Per $M$. ulcerans il serbatoio è sconosciuto, mentre si aggiungono il terreno e altri animali per $M$. fortuitum.

M. marinum, che ha una struttura antigenica in parte comune con $M$. tuberculosis, abitualmente presente nelle acque salate e negli organismi marini (colonizza la superficie dei pesci e dei crostacei), ma isolato anche in piscine e acquari, è responsabile di un'infezione umana che fa seguito ad un trauma minimo, avvenuto in piscina $o$ in acquari (addetti alla manutenzione) (6) o più suggestivamente alla puntura di pesce o morso di crostaceo (esposizione professionale ed hobby della pesca) (6).

Le lesioni appaiono sempre confinate alle estremità e precisamente ai tessuti superficiali meno caldi. L'incubazione è di solito di due-tre settimane, ma sono stati descritti casi con brevissima incubazione di cinque giorni, e alcuni con incubazione sino a 270 giorni (6).

Le lesioni istopatologiche vanno da una suppurazione franca a granulomi organizzati, evoluti a seguito di un processo caratterizzato da papulonoduli di colore blu-rosso vinoso (swimming pool granuloma o fish tank granuloma). Talvolta le lesioni nodulari, suppurate e progredite sino allo stato di ulcere, si dispongono lungo i vasi linfatici efferenti, associandosi a linfangite, così come avviene nella sporotricosi (4).

Meno frequentemente $M$. marinum è responsabile di lesioni più profonde quali tenosinoviti e artriti. È stato descritto anche un caso di sclerocheratite e più recentemente è stato riportato dalla letteratura una infezione miositica dell'avambraccio (8).

\section{CASO CLINICO}

T.M. uomo di 31 aa, con hobby della pesca, si punge accidentalmente con la spina di un pesce di scoglio al terzo dito della mano destra. A distanza di tre settimane, nella sede della puntura, sviluppa una lesione nodulare-eritematosa che ben presto si ulcera e secerne pus (figura I).

Dopo qualche settimana si assiste alla comparsa di una seconda lesione, seguita da numerose altre, con aspetto simile alla prima, di dimensioni variabili, lungo il decorso dei linfatici afferenti dell'avambraccio (figure II e III), alcune spontaneamente dolenti e visibili altre solo palpabili e con disposizione satellitare a quelle più voluminose.

A due mesi dall'evento acuto si sottopone ad osservazione sanitaria: viene eseguita agobiopsia 


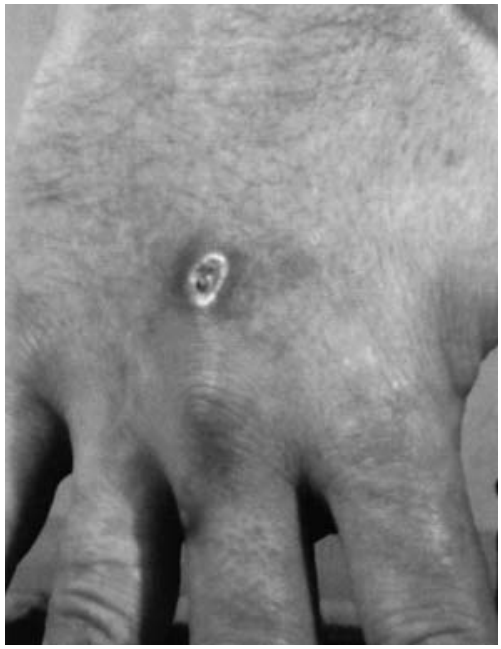

Figura I. Lesione nodulare eritematosa ulcerata secernente

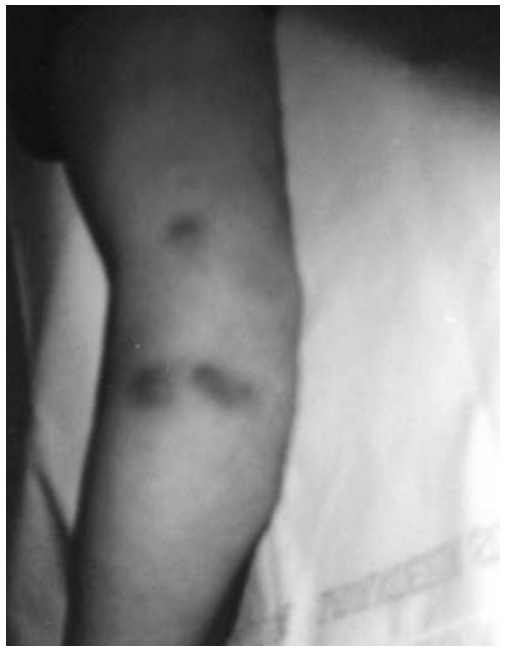

Figura II. Lesioni nodulari eritematose lungo il decorso dei vasi linfatici

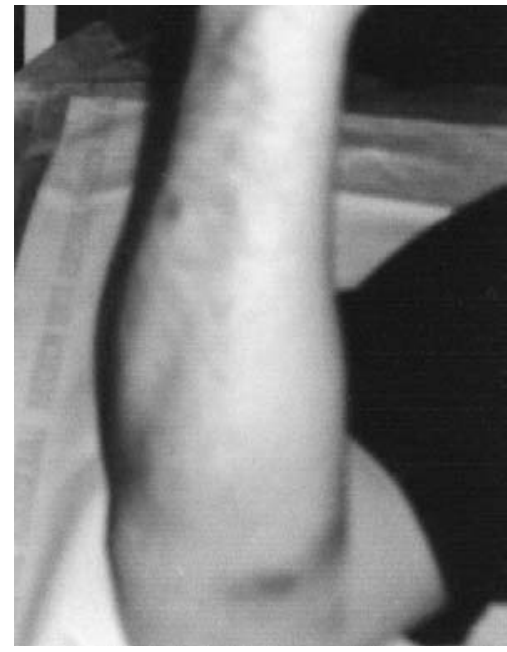

Figura III. Lesioni nodulari eritematose lungo il decorso dei vasi linfatici della cute e agoaspirato su materiale purulento che consentono di porre diagnosi di dermatite granulomatosa di probabile natura infettiva.

Il sospetto clinico di una etiologia Mycobatterica non viene confermato da una prima indagine microbiologica.

Normali sono pure gli esami ematochimici e quello emomorfocitometrico.

$\mathrm{Si}$ avvia terapia con Doxiciclina (100 mg per os bid per quattro settimane) senza alcun beneficio, mantenendosi le lesioni per lo più invariate salvo alcune con tendenza all'ulcerazione. Un esame ecografico conferma la presenza di lesioni nodulari, la più voluminosa delle quali, non evidente ad un precedente esame, si affonda nel piano cutaneo per circa $8 \mathrm{~mm}$, poggiando sulla guaina muscolare.

$\mathrm{Si}$ asporta, quindi, un intero nodulo (figura IV) che viene inviato al Servizio di Virologia e Microbiologia del P.O. dell'Annunziata di Cosenza.

In data 10.04.01 il nodulo viene suddiviso in 5 piccoli pezzi che sono sottoposti alle seguenti indagini:

$>$ striscio su vetrini per effettuare vari tipi di colorazioni (Ziehl-Neelsen, Auramina, Gram);

$>$ semina in thioglicolato per la ricerca di germi comuni e miceti;

$>$ semina in 2 brodi MGIT di cui uno viene mantenuto a Temperatura ambiente e un altro viene incubato a $37^{\circ} \mathrm{C}$ in presenza del $5 \%$ di $\mathrm{CO}_{2}$.

Dopo 7 giorni dalla semina compare una positività nel brodo MGIT mantenuto a T.a. e l'esame microscopico mostra la presenza di rarissimi bacilli alcool acido resistenti. Si procede allora ad un arricchimento trasferendo il tutto in un altro brodo MGIT che viene nuovamente lasciato a T.a.
Il 02.05.01 il MGIT mostra una nuova positività per cui si iniziano le ricerche volte alla evidenziazione di bacilli alcool acido resistenti nonché alla loro tipizzazione. In particolare, una volta eseguito l'esame microscopico che mostra bacilli ZiehlNeelsen e Auramina positivi con caratteristiche bandeggiature (figura V), vengono seminati con il sedimento risospeso in tampone fosfato a $\mathrm{pH} 6.8$ n. 4 tubi di Lowenstein-Jensen di cui 2 sono posti a $37^{\circ} \mathrm{C}$ ( 1 al buio e 1 alla luce) e 2 a T.a. ( 1 al buio e 1 alla luce).

I risultati (crescita al $16^{\circ} \mathrm{C}$ giorno, temperatura ottimale $25^{\circ} \mathrm{C}$, colonie fotocromogene, catalasi a $68^{\circ} \mathrm{C}$ positive, idrolisi del Tween 80 positive) indirizzano verso un ceppo di Mycobacterium marinum.

L'isolato è quindi inviato al laboratorio di Microbiologia dell'Ospedale Careggi di Firenze per la conferma in HPLC. Il profilo ottenuto (figura VI) conferma la diagnosi.

$\mathrm{Si}$ avvia pertanto la terapia con Rifampicina (300 $\mathrm{mg}$ bid) ed Etambutolo (500 mg tid).

A tre settimane si assiste a miglioramento clinico con riduzione volumetrica delle lesioni.

A dodici settimane si ha guarigione completa e si interrompe la terapia.

\section{CONCLUSIONI}

Il caso, per le manifestazioni cliniche presentate in seguito a trauma minimo della cute per puntura di spina di pesce, per il decorso a carattere cronico delle risposte istopatologiche, per la variabilità delle lesioni (papulo-noduli, suppurazioneulcerazione, granulomi) e per la disposizione lungo i vasi linfatici, è patognomonico di infezione sporotricoide.

Già il dato anamnestico, rilevante il fattore di rischio esposizionale, differenzia la malattia da noi osservata dalla Sporotricosi (agente Sporotrix 


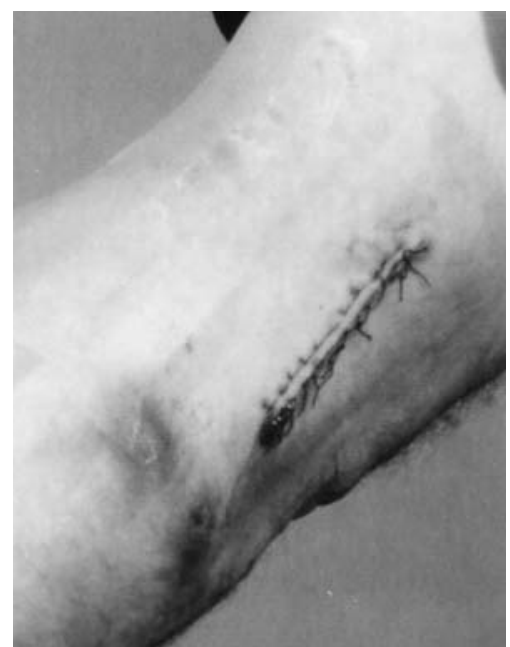

Figura IV. Esito asportazione chirurgica lesione granulomatosa avambraccio destro

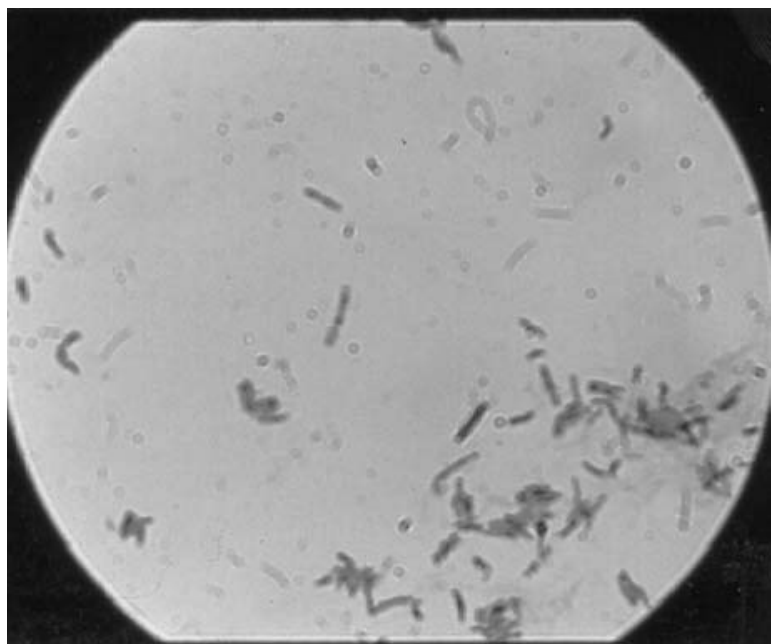

Figura V. Bacilli alcool-acido resistenti con bandeggiatura

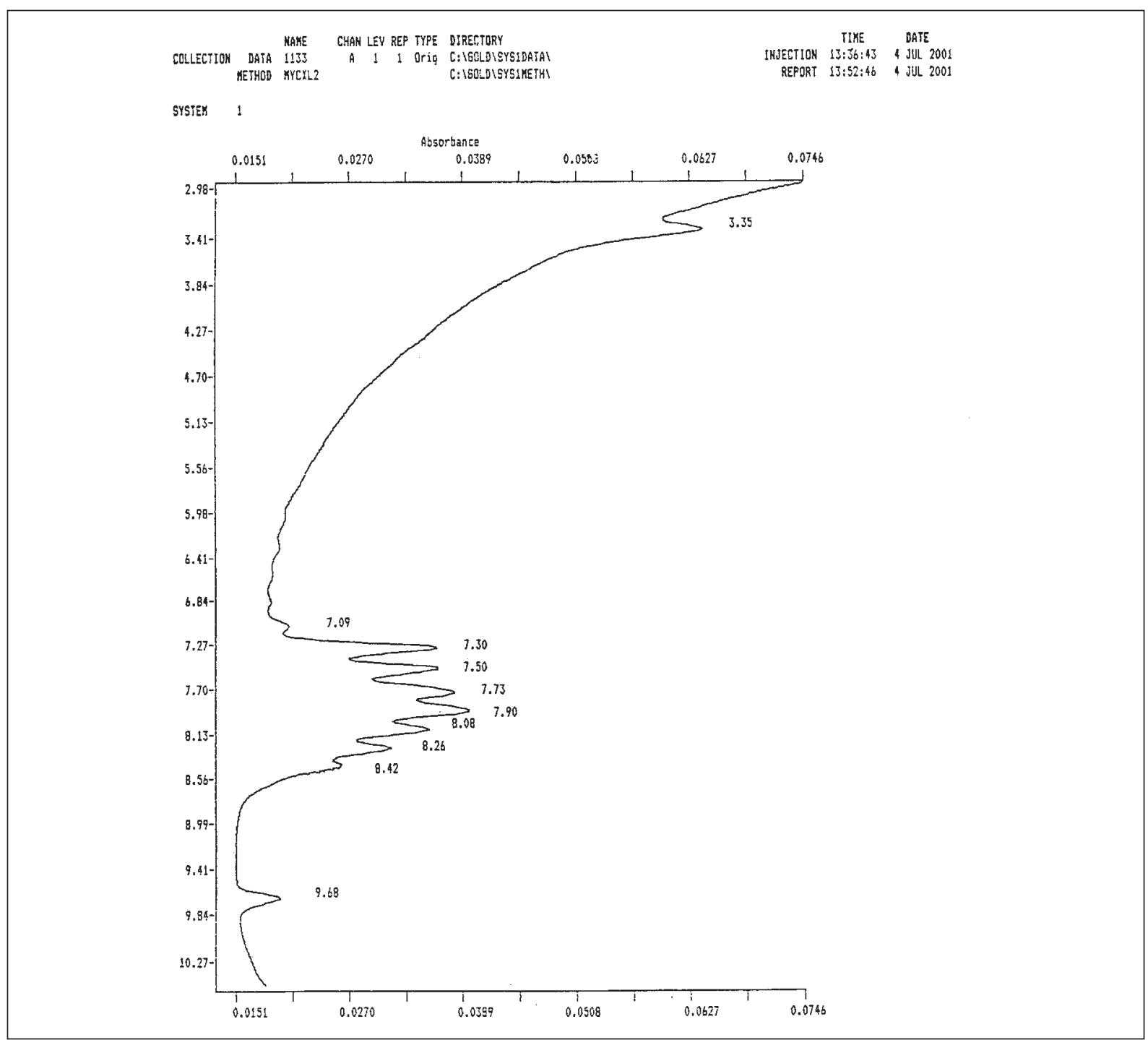

Figura VI. Profilo di M, marinum in HPLC

Scenkii), tuttavia la conferma di tale differenziazione è basata sull'evidenza del risultato colturale del pezzo bioptico, che permette l'ulteriore distinzione dell'etiologia da $M$. marinum dagli altri micobatteri patogeni per la cute.

L'esame istologico precisa il danno tissutale come 
granuloma e infezione granulomatosa.

La ricerca batterioscopica ha dato esito negativo, come di frequente, in accordo con i dati della letteratura. L'esame colturale per germi comuni e le ricerche, mediante PCR, del genoma del Mycobacterium tuberculosis complex, condotte inizialmente su materiale biologico secreto (pus) hanno dato esito negativo. La ricerca colturale rivolta ai micobatteri eseguita su un intero pezzo bioptico ha dato esito positivo per Mycobacterium marinum.

Il trattamento riconosce diverse opzioni terapeutiche costituite dalla somministrazione di antibiotici e di agenti antitubercolari, e in alcuni casi dall'escissione chirurgica delle lesioni.

L'utilizzo della Minociclina, enfatizzata da alcuni, nel nostro caso non ha dato risultati per cui, tra le opzioni terapeutiche consigliate, Trimetophimsulfametossazolo, Claritromicina o Rifampicina più Etambutolo (7), è stata scelta quest'ultima con risposta terapeutica già alla terza settimana e guarigione completa alla dodicesima settimana.

Secondo alcuni dati, sarebbe bene utilizzare Cotrimoxazolo nelle forme più superficiali e l'associazione Rifampicina-Etambutolo nelle forme profonde (7).

La terapia chirurgica, peraltro da noi attuata su un intero nodulo, a scopo diagnostico, andrebbe riservata alle forme resistenti ai farmaci (1) ma, seppur fattibile per le lesioni distinte e circondate da ampie zone di cute sana, non è proponibile per lesioni multiple e contigue, perché vaste aree cutanee andrebbero asportate, con difficoltà di chiudere esteticamente i lembi su superfici visibili.

\section{RINGRAZIAMENTI}

Si ringrazia il Dott. Enrico Tortoli per la collaborazione nella esecuzione dell'HPLC.

\section{BIBLIOGRAFIA}

1. Amrani M, Renoirte P, Pieron P, Six C. Skin lesion due to Micobacterium marinum: surgical ablation, apropos of case of false paronychia. Acta chir belg 1991; 91 (5): 265-8.

2. Falkinham JO. Epidemiology of infection by nontubercolous mycobacteria. Clin Microbiol 1996; 34: 1840-2.

3. Gbery IP, Djeaha D, Yobouet P, Aka B, Kanga JM. Atypical micobacterial skin infectious. Sante 1996; 6 (5): 317-22.

4. Hofbauer GF, Burg G, Nestle FO. Sporotrichoid infection with $M$. marinum: successful therapy with oral tetracycline administration. Hautarzt 2000; 51 (5): 349-52.

5. Holmes GF, Harrington SM, Romagnon MJ, Merz WG. Recurrent, disseminated M. marinum infection caused by the same genotypically defined strain in an immunocompromised patient. J Clin Microbiol 1999;
37 (9): 3059-61.

6. Jernigan JA, Farr BM. Incubation period and sources of exposure to cutaneous $M$. marinum infection: case report and review of the literature. Clin Infect Dis 2000; 31 (2): 439-43.

7. Leuenberger R, Bodmer T. Clinical presentation and therapy of Mycobacterium marinum infection as sees in 12 cases. Dtsch med wochenschr 2000; 125 (1-2): 7-10.

8. Vasanthi A, Scott M, Carol S. Mycobacterium marinum myositis. Infect Dis Clin Practice 2000; 9 (9): 376-8.

9. Wolinsky E, Rynearson TK. Mycobacteria in soil and their relation to disease-associated strains. Am Rev Resp Dis 1968; 97: 1032-7.

\section{Paolina Cavalcanti}

Servizio di Virologia e Microbiologia

Presidio Ospedaliero Annunziata

Via Zara; 87100 Cosenza

Tel.: 0984 681822;

e-mail: batteriologia@libero.it 